\title{
BMJ Open Effectiveness of technology-based interventions in detection, prevention, monitoring and treatment of patients at risk or diagnosed with mild cognitive impairment: protocol for a systematic review
}

\author{
Jordi Martínez-Soldevila, ${ }^{1,2,3}$ Roland Pastells-Peiró (D) , ,2,3 \\ Carolina Climent-Sanz (1) , , ${ }^{1,3}$ Gerard Piñol-Ripoll, ${ }^{4}$ Mariona Rocaspana-García, , ${ }^{1,2}$ \\ Montserrat Gea-Sánchez (i) 1,2,3
}

To cite: Martínez-Soldevila J, Pastells-Peiró R, ClimentSanz C, et al. Effectiveness of technology-based interventions in detection, prevention, monitoring and treatment of patients at risk or diagnosed with mild cognitive impairment: protocol for a systematic review. BMJ Open 2021;11:e045978. doi:10.1136/ bmjopen-2020-045978

- Prepublication history for this paper is available online. To view these files, please visit the journal online (http://dx.doi. org/10.1136/bmjopen-2020045978).

Received 23 0ctober 2020 Accepted 29 July 2021

Check for updates

(c) Author(s) (or their employer(s)) 2021. Re-use permitted under CC BY-NC. No commercial re-use. See rights and permissions. Published by BMJ.

For numbered affiliations see end of article.

Correspondence to Dr Carolina Climent-Sanz; carol.climent@udl.cat

\section{ABSTRACT}

Introduction The gradual changes over the decades in the longevity and ageing of European society as a whole can be directly related to the prolonged decline in the birth rate and increase in the life expectancy. According to the WHO, there is an increased risk of dementia or other cognitive disorders as the population ages, which have a major impact on public health. Mild cognitive impairment (MCl) is described as a greater than expected cognitive decline for an individual's age and level of education, but that does not significantly interfere with activities of daily living. Patients with $\mathrm{MCl}$ exhibit a higher risk of dementia compared with others in the same age group, but without a cognitive decline, have impaired walking and a $50 \%$ greater risk of falling.

The urban lifestyle and advent of smartphones, mobility and immediate access to all information via the internet, including health information, has led to a totally disruptive change in most general aspects.

This systematic review protocol is aimed at evaluating the effectiveness of technology-based interventions in the detection, prevention, monitoring and treatment of patients at risk or diagnosed with $\mathrm{MCl}$.

Methods and analysis This review protocol follows the recommendations of the Preferred Reporting Items for Systematic Review and Meta-Analysis Protocols reporting guidelines. The search will be performed on MEDLINE (PubMed), CENTRAL, CINAHL Plus, ISI Web of Science and Scopus databases from 2010 to 2020. Studies of interventions either randomised clinical trials or pre-post non-randomised quasi-experimental designs, published in English and Spanish will be included. Articles that provide relevant information on the use of technology and its effectiveness in interventions that assess improvements in early detection, prevention, follow-up and treatment of the patients at risk or diagnosed with $\mathrm{MCl}$ will be included. Ethics and dissemination Ethics committee approval not required. The results will be disseminated in publications and congresses.
Strengths and limitations of this study

- Criteria and data points valid for a systematic review and meta-analysis were followed.

- Databases and search strategies were selected and designed by an expert.

- A blinded peer review will be conducted using a rigorous and consistent set of criteria.

- The effectiveness and use of technologies are questioned and more research is needed.

\section{INTRODUCTION}

The WHO indicated that the definition of 'older persons' or 'elderly' is controversial; as each country has established different criteria for categorising a person within a chronological age. Most of the world's advanced countries have accepted that the term 'older person' should correspond to a person over 65 years of age; however, this criterion cannot be applied globally, as it would not correspond to the African countries. In contrast, the United Nations Organisation established a standard numerical criterion, which categorises people over 60 years of age as elderly. ${ }^{1}$

The gradual change over the decades in the longevity and ageing of European society as a whole can be directly linked to the prolonged decline in the birth rate and increase in life expectancy. The data show that this trend will be perpetuated over a long period..$^{23}$

Furthermore, future projections of the European Statistical Office (Eurostat) ${ }^{2}$ have indicated that this ageing trend will increase, with people aged 65 years and over accounting for $29.1 \%$ of the total population in 2060 and 
indicated a significant increase in the population aged over 80 years, which will reach a percentage of $14.6 \%$ of the total population in 2100. There are two main factors directly related to this trend in the European regions: (1) the dynamics of fertility and life expectancy at birth and (2) improvements in health and quality of life standards. ${ }^{3}$

Another report published by Eurostat during 2015 highlighted the increase in the percentage of people living alone across Europe. Improvements in technology, communication, resources, infrastructure and environmental factors benefit and possibly facilitate life in solitude.

The southern European countries, such as Cyprus, Portugal, Greece and Spain show a lower percentage of older people living alone compared with their European neighbours. ${ }^{4}$

Specifically, in Spain, as per the data released by the National Statistics Institute in the 'Continuous Household Survey, 2017' there are 4687.4 billion people living in single-person households, representing $41.8 \%$ of all Spanish households. Furthermore, the number of men and women living in single-person households is different. Approximately 1.4 million single-person households are inhabited by women and around 0.6 million by men. In addition, the study provides data on cohabitation in old age, a situation that differs between men and women. Most men live with a partner (spouse, partner with or without children or others), representing $14.7 \%$ of men who live alone, while $29.3 \%$ of women live alone because of the early death of partner. In Spain, the total number of elderly persons living alone in their homes has reached $22.9 \%$. This factor, in correlation with the health problems associated with ageing, the lack of a social support network and the feeling of loneliness could generate serious problems leading to a loss of well-being and drastic reduction in the quality of life self-perceived by the elderly person. ${ }^{5-7}$

In addition, as the population ages, there is an increased risk of cognitive impairment of varying intensity, which has a significant public health impact according to the WHO. ${ }^{8}$ Mild cognitive impairment (MCI) is described as a cognitive decline that is greater than expected for an individual's age and level of education, but does not significantly interfere with activities of daily living and therefore, does not require early institutionalisation. Patients with MCI have a higher risk of dementia compared with others in the same age group, but without cognitive decline, show gait disturbances (such as decreased walking speed) and a $50 \%$ greater risk of experiencing falls. ${ }^{9}$

Caregivers and families are also affected especially, when the patient with dementia is in a moderate or advanced phase. This overload is one of the main reasons for the institutionalisation of patients. Hence, we propose to use monitoring to reduce this load described in professional caregivers and families.

According to a report by the Spanish Society of Geriatrics and Gerontology, no studies have estimated the economic impact of International Marketing and
Communication, SA. ${ }^{10}$ Considering that MCI may be a pre-dementia stage, the direct and indirect economic burden will increase with the patient's age. Alzheimer's disease $(\mathrm{AD})$ is the most common cause of dementia. The Organisation for Economic Cooperation and Development statistics showed that in 2018 there were 9.1 million patients over 60 years of age diagnosed with $\mathrm{AD}$ in the European Union (out of a total population of 513 million), and this number is expected to increase by $60 \%$ by 2040 reaching 14.3 million people with $\mathrm{AD} .{ }^{11}$ In addition, the health cost per patient is estimated to be around $20.000 €$ per year. ${ }^{12}$ As for the USA, it is estimated that AD alone will affect more than 16 million people in 2050, at a cost of more than US $\$ 1.1$ billion. ${ }^{13}$

In Spain, the costs associated with cognitive impairment, dementia and AD in 2014 are estimated to be approximately $€ 30000$ per patient. An important factor to be considered is that since 2010 the health costs associated with dementia have increased by $35 \%$ and this increase is mainly in the developed countries. ${ }^{14}$

Healthcare has long used advanced technological equipment to measure and better understand the patient's health status. In the past, researchers have focused on developing increasingly sensitive and accurate measurement devices for hospital use. The interpretation of results and diagnosis was left to expert physicians, who took the responsibility of decisions and treatment. ${ }^{15}$

However, the ongoing paradigm shift in healthcare requires refocusing care and moving it into a proactive and preventive perspective, looking for ways to keep people healthy by influencing their lifestyle and early detection of complications. The changes produced in recent years in the demand, management and financing of the health system were because of the circumstances that the system itself is going through to offer the established portfolio of services, its viability in the future and consequences and problems produced by the economic crisis. Hence, health professionals encouraged and educated patients to take control of their own healthcare process; thus, involving and empowering them. Over the years, it has been established that prevention only works when patients are actively involved in the process. The increasing amount of self-care and self-management of their own pathologies or disease processes has reduced hospital stays and frequent visits of the patients.

Demographic changes, including the ageing of society and consequent increase in the demand for healthcare, have made it essential for managers and professionals to seek alternatives and improvements in the efficiency of care provided at the individual and home level, always with a dual purpose: to reduce economic costs and increase the quality of services. ${ }^{1617}$

Technological advances have made these changes possible, as patients can now have solutions related to their health without the direct interference of medical staff.

Healthcare should benefit much more from proactive and discreet monitoring of healthy people and those most 
at risk of illness (eg, older adults) through sensors built into their environment. This contributes to increased patient safety, early detection, prevention of illness, improved monitoring and treatment; therefore, reduced costs for the corresponding care provided. ${ }^{17}$

The urban lifestyle and advent of smart phones, mobility and immediate access to all information via the internet, including health information, has led to a totally disruptive change in most aspects of the daily life of the population with a certain degree of digital literacy. Hence, the health and welfare market has been subjected to a great impact. Customers are demanding an improvement in their quality of life and are willing to invest their resources in new technologies and consumer services that will generate an improvement in their daily lives. ${ }^{18}$

The potential and market niche for smart health products, solutions and services is substantial and growing rapidly and exponentially. Specific numbers detailing the size of the market or directly supporting growth expectations are not easy to calculate, as the quantitative data entering the field of smart health are rarely tracked or labelled as such in formal statistics. However, several calculations allow us to estimate the current size of the market and growth expectations in a generic way. Despite the difficulties associated with data availability, a study on the market deployment of internet of things solutions shows that the global smart health market is expected to grow by more than $19 \%$ annually. ${ }^{19}{ }^{20}$ The much more expansive internet of people or internet of everything market, which in the near future, will include machineto-machine, machine-to-person and person-to-person communications with almost infinite potential that cannot be ignored. ${ }^{21}$

The intelligent systems used for control and decisionmaking in healthcare are based on a variety of technologies that collect general and specific data through sensors and perform an in-depth analysis. The scans are performed by a combination of carefully chosen sensors, which probe various parameters, such as physiological parameters, habitual behaviour, activity level, location, among others. Perception sensors are specifically suited for this purpose, but their use is not common. Currently, in the field of healthcare, the most widely used devices for detecting and measuring variables, work by means of added cables, and clothing are considered as intelligent clothing because of their characteristics, performance and functionalities provided by the incorporated fabrics.

Currently, several technologies are available in the market that increase the sense of security and independence of the elderly. However, all these technologies cover only limited areas of the basic activities of daily life. Human functioning reflects a complex interaction between a person's health status and contextual factors. Therefore, a holistic view of daily activities using discreet surveillance technologies is needed, allowing for a longterm trend analysis of the routines of the elderly in the context of their individual life situation and environmental impact. ${ }^{22}$
The practical implications of this review essentially aim to identify existing interventions that use technologies to determine their effectiveness for the detection, prevention, monitoring and treatment of patients at risk or diagnosed with MCI.

\section{OBJECTIVE}

The aim of this systematic review will be to evaluate the effectiveness of technology-based interventions in the detection, prevention, monitoring and treatment of patients at risk or diagnosed with MCI.

\section{METHODS AND ANALYSIS \\ Design}

The protocol for this review follows the recommendations of the Preferred Reporting Items for Systematic Reviews and Meta-Analyses for Protocols (PRISMA-P) reporting guidelines. The PRISMA tool will be used to conduct the intervention review. ${ }^{23}$

\section{Inclusion criteria \\ Participants}

All studies that are conducted with persons aged 65 years or older who are at risk or diagnosed with MCI, and who are living independently in the community, alone, with family members or with caregivers will be included in this review; regardless of their sex, ethnicity, socio-economic or socio-cultural background.

\section{Study design}

Intervention studies will be included in the review, randomised clinical trials in any of their designs or prepost non-randomised quasi-experimental designs, as these are the designs that are mostly used in the field of medical informatics. ${ }^{24}$

\section{Interventions}

All the studies using information and communication technologies, such as pedometers, activity of daily living, such as exercise, heart rate or sleep monitoring, intelligent movement detection, devices or any other device or tool, to send, store and process information relevant to the prevention, detection, monitoring and treatment of patients at risk or diagnosed with MCI will be included in this systematic review.

\section{Results}

Articles will be included that provide relevant information on the use of technology and its effectiveness in interventions that assess improvements in relation to early detection, prevention and treatment of patients at risk or diagnosed with MCI.

Sources of information, management and data collection

The following databases: MEDLINE (PubMed), CENTRAL, CINAHL Plus, ISI Web of Science and Scopus 
from 2010 to 2020, for the published articles in English and Spanish will be searched.

The search will be restricted to studies published between 2010 and 2020. The literature supports the premise that technology is advancing rapidly and that, therefore, including studies published before 2010 would yield obsolete results for our research objective. ${ }^{25} 26$

We will additionally perform the following two actions to ensure that any relevant results are not missed: (1) A search on Google Scholar, considering the characteristics and shortcomings of the search engine in the systematisation of the syntax of the search strategy. (2) A manual search through the references of the most relevant studies. The search strategy for MEDLINE (PubMed) will be designed, following the recommendations of the Peer Review of Electronic Search Strategies statement. ${ }^{27}$ Once the reviewers have reached a consensus, the strategy will be transferred to the aforementioned databases, adapting it to the particularities of each of the databases (syntax and proximity operators). The references obtained will be downloaded in the EndNote V.X9.2 reference manager software, which will also be used for the data deduplication process. ${ }^{28}$

The initial search strategies proposed for MEDLINE (PubMed) and Scopus are presented in tables 1 and 2, respectively.

\section{Selection process of studies}

The results obtained after the deduplication process will be exported to Covidence, a web-based literature manager software. ${ }^{29}$ Through this platform, first, screening by title and abstract will be performed by two independent reviewers, according to the inclusion and exclusion criteria with the premises of relevant, irrelevant or doubt. At this stage the reviewers will explain the reason for exclusion of the article. When there is no consensus and in case of doubts, a third reviewer in discussion with the other two reviewers will make the decision. Once this screening by title and abstract is completed, the full texts of the articles will be searched, which will also be entered into the Covidence software. During the full-text screening, the reviewers will record the operationalisation of the variables in a codebook. Two investigators will independently review $25 \%$ of the randomly selected articles and $25 \%$ of the operationalisation of all variables written in the codebook. A third investigator will arbitrate in case of discrepancies. The results for each item will be checked to ensure that they are classified in the same category. Subsequently, the results will be analysed using the Kappa index formula through the IBM SPSS Statistics software (version 25), showing the level of agreement between the reviewers for included and excluded studies.

\section{Evaluation of methodological quality and control of risk of bias}

The studies included in the review will be analysed by the appropriate tools for their design. Thus, for randomised clinical trials, the Cochrane Reviews risk of bias 2 tool will

\begin{tabular}{|c|c|c|}
\hline Concepts & PubMed search strategy & $\begin{array}{l}\text { Search } \\
\text { results }\end{array}$ \\
\hline Technology & 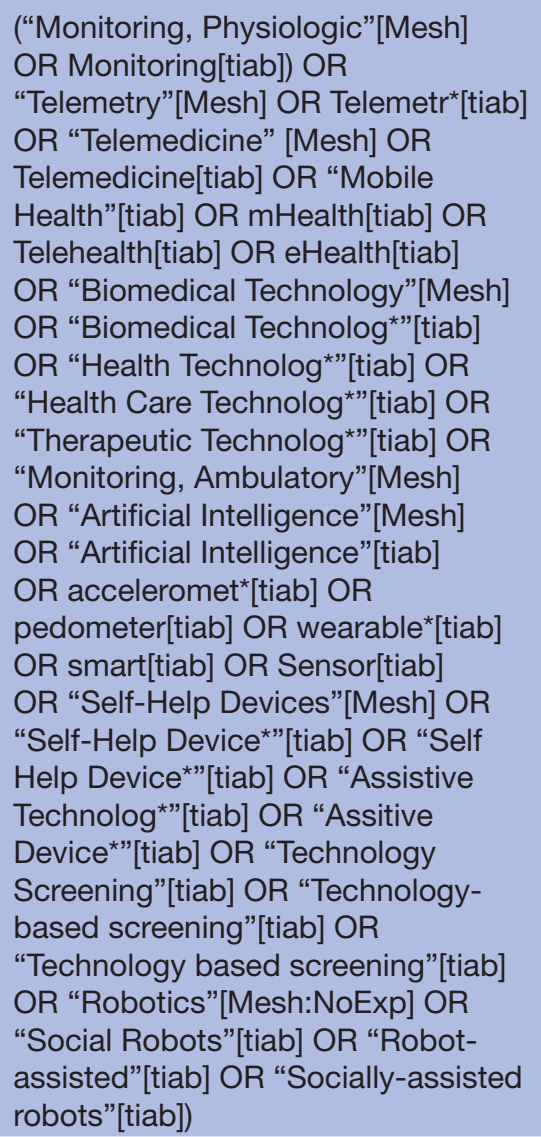 & $\# 1$ \\
\hline $\begin{array}{l}\text { Mild } \\
\text { cognitive } \\
\text { impairment }\end{array}$ & $\begin{array}{l}\text { (“Cognitive Dysfunction”[Mesh] } \\
\text { OR “Cognitive Dysfunction*”[tiab] } \\
\text { OR “Cognitive Decline*”[tiab] OR } \\
\text { "Mental Deterioration" OR “Mild } \\
\text { Cognitive Impairment”[tiab] OR “Mild } \\
\text { Cognitive Disorder"[tiab] OR "Mild } \\
\text { Neurocognitive Impairment”[tiab] OR } \\
\text { "Mild Neurocognitive Disorder"[tiab]) }\end{array}$ & $\# 2$ \\
\hline Total & \# 1 AND \# 2 & \# 3 \\
\hline
\end{tabular}

be used to assess the risk of bias, and for pre-post nonrandomised experimental studies, the checklist for quasiexperimental studies will be used. The PEDro scale ${ }^{30}$ will be used to assess the methodological quality of pre-post non-randomised trials. The PEDro scale consists of 10 items that permit the evaluation of ${ }^{30}:(1)$ random allocation, (2) concealed allocation, (3) similarity at baseline, (4) subject blinding, (5) therapist blinding, (6) assessor blinding, (7) $>85 \%$ follow-up for at least one key outcome, (8) intention-to-treat analysis, (9) betweengroup statistical comparison for at least one key outcome and (10) point and variability measures for at least one key outcome.

The certainty of the evidence will be rated according to the GRADE systematic approach. We will use the methodology proposed in the GRADE evidence profile table 
Table 2 Scopus search strategy

\begin{tabular}{|c|c|c|}
\hline Concepts & Scopus search strategy & $\begin{array}{l}\text { Search } \\
\text { results }\end{array}$ \\
\hline Technology & $\begin{array}{l}\text { TITLE-ABS-KEY (Monitoring OR } \\
\text { Telemetr* OR Telemedicine OR } \\
\text { "Mobile Health" OR mHealth } \\
\text { OR Telehealth OR eHealth OR } \\
\text { ((Biomedical OR Health OR } \\
\text { Therapeutic OR Assistive OR } \\
\text { Screening) W/3 (Technolog* OR } \\
\text { Technology-Based OR "Technology } \\
\text { based")) OR "Artificial Intelligence" } \\
\text { OR acceleromet* OR pedometer } \\
\text { OR wearable* OR smart OR Sensor } \\
\text { OR "Self-Help Device" OR "Self } \\
\text { Help Device*" OR "Assistive } \\
\text { Technolog*" OR "Assitive Device*" } \\
\text { OR "Social Robots" OR "Robot- } \\
\text { assisted" OR "Socially-assisted } \\
\text { robots") }\end{array}$ & $\# 1$ \\
\hline $\begin{array}{l}\text { Mild } \\
\text { cognitive } \\
\text { impairment }\end{array}$ & $\begin{array}{l}\text { TITLE-ABS-KEY (“Cognitive } \\
\text { Dysfunction"” OR "Cognitive } \\
\text { Decline*" OR "Mental } \\
\text { Deterioration" OR "Mild Cognitive } \\
\text { Impairment" OR "Mild Cognitive } \\
\text { Disorder" OR "Mild Neurocognitive } \\
\text { Impairment" OR "Mild } \\
\text { Neurocognitive Disorder") }\end{array}$ & $\# 2$ \\
\hline Total & \# 1 AND \# 2 & \# 3 \\
\hline
\end{tabular}

(new version) of the Handbook 'manual grade: Grading of Recommendations Assessment, Development and Evaluation' proposed by the GRADE working group. ${ }^{31}$

\section{Data extraction and synthesis}

The data to be extracted will be adapted according to the recommendations of the Cochrane Handbook for Systematic Reviews of Interventions. ${ }^{32}$ The following data will be extracted: (1) Identification data of each of the studies: authors, title, country of publication, year of publication and funding of the study. (2) Data concerning the design of the study: design, intervention used, type of participants and technology used. (3) Data concerning the results: main results, limitations and conclusions. First, we will detect missing data and classify them into three categories: (1) completely random, (2) random and (3) nonrandom. To avoid loss of information, we will perform an estimation by imputing the missing data to each test arm expressing the degree of deviation, and replacing the data with predicted values from the collected data. ${ }^{33}$

The extracted data will be peer reviewed, establishing a first pilot to check if the flow in the extracted information is correct.

Clinical heterogeneity of the studies included in this systematic review will be assessed by analysing similarity in participants, setting, interventions and outcome measures. The variability in study design and risk of bias will be examined to evaluate methodological heterogeneity. The $\chi^{2}$ test will be used to calculate the statistical heterogeneity, setting the level of significance at 0.05 . In the presence of substantial heterogeneity $(>80 \%)$ the pooling of studies will not be implemented.

\section{Patient and public involvement}

The data will be obtained from previously published research. Patients, public and private institutions and other entities are not involved in the development of the research question, outcome measures or study design. This research will not involve the participation of patients.

\section{DISCUSSION}

Ageing is an intrinsic reality that is marked by cellular ageing and genetic programming. ${ }^{34}$ Human beings must face this scenario with a certain foresight and thus, be able to adapt correctly to the social, economic and health needs that appear throughout the process of ageing. Similarly, there are theories of social and psychosocial ageing reporting the changes that occur with the passage of time and the evolution of the individual. ${ }^{35}$

However, we must look at this situation not only at the individual level, but also at the collective level. The increase in life expectancy in developed countries is causing their population pyramids to age, leading to a new reality that requires an exhaustive assessment and evaluation of future sociodemographic scenarios by experts in the field. People structure the different environments or spheres that surround them according to their needs and values, which can vary according to the moment in the life cycle where they are. The adaptation to the natural and constant changes that are conceived in the different stages of life, physical and psychic level, state of health, quality of life, family, social, religious and economic context will finally mark the needs that each individual can present and the support that they can have or need at the end of their life cycles. ${ }^{36} 37$

Some researchers have performed interventions where they evaluated the effectiveness and use of new technologies in people over 65 years of age, although these interventions are not directly related to our study objective, since their inputs were directed to the reduction of loneliness, social isolation, chronic diseases and disabilities in general. These dissertations can support our study objective, as they showed promising efficacy. However, the authors emphasised the need for much more comprehensive intervention designs and evaluations. ${ }^{38} 39$

Therefore, we considered the need to review the existing scientific literature on the use of technology in elderly people at risk or diagnosed with MCI. The main objective of our research is to improve the monitoring and treatment of patients at risk or diagnosed with MCI, to achieve a better prognosis through closer monitoring and application of early therapeutic strategies. Hernández et al conducted a cross-sectional descriptive correlation study where they collected and analysed data using technology and reported that through acoustic variables and 
verbal fluency, early detection and monitoring of MCI could be achieved. ${ }^{40}$

The results of this review will facilitate the identification of interventions where technology is used, for the detection, prevention, follow-up and treatment of patients at risk or diagnosed with MCI. Furthermore, this study will evaluate the methodological quality of the published research, which will enable to review and offer new perspectives of use of the sensorial technology in this specific health problem.

\section{ETHICS AND DISSEMINATION}

Ethics committee approval is not required for this protocol and subsequent systematic review. The main objective is to extract and summarise the results of studies that meet the inclusion criteria.

The results of the study and additional data will be disseminated through publications and participation in scientific conferences and workshops. A communication will be sent to the Healthio 2021.

\section{Author affiliations \\ ${ }^{1}$ Department of Nursing and Physiotherapy, University of Lleida, Lleida, Catalonia, Spain \\ ${ }^{2}$ Health Care Research Group (GRECS), Lleida Institute for Biomedical Research Dr. Pifarré Foundation, IRBLLEIDA, Lleida, 25198, Catalonia, Spain \\ ${ }^{3}$ Grup d'Estudis Societat, Salut, Educació i Cultura (GESEC), Universitat de Lleida, Lleida, Catalonia, Spain \\ ${ }^{4}$ Cognitive Disorders Unit, Clinical Neuroscience Research, Institut de Recerca Biomèdica de Lleida Fundació Doctor Pifarré, Lleida, Catalonia, Spain}

Twitter Jordi Martínez-Soldevila @JmartinezSol, Carolina Climent-Sanz @Carol_ Climent and Montserrat Gea-Sánchez @MontserratGea

Acknowledgements The researchers would like to thank the Health Care Research Group (GRECS) of the Biomedical Research Institute of Lleida, Santa Maria University Hospital of Lleida and the University of Lleida for their collaboration.

Contributors The systematic review protocol was elaborated and considered by JM-S, RP-P, MG-S and MR-G who participated in the review of the manuscript by providing the methodological aspects and contributed to the search strategy. CC-S and GP-R participated in the supervision, quality control and guaranteed the excellence of the protocol.

Funding This paper is a result of the NEXTPERCEPTION project (www. nextperception.eu), which is jointly funded by the European Commission and national funding agencies under the ECSEL joint undertaking (grant agreement: 876487). This research was funded by the Agencia Estatal de Investigación (AEI/10.13039/501100011033) Ministry of Science and Innovation (MICINN) (PCI2020-112270) CERCA Programme/Generalitat de Catalunya. IRBLleida Fundació Dr. Pifarré.

Competing interests None declared.

Patient and public involvement Patients and/or the public were not involved in the design, or conduct, or reporting, or dissemination plans of this research.

Patient consent for publication Not required.

Provenance and peer review Not commissioned; externally peer reviewed.

Open access This is an open access article distributed in accordance with the Creative Commons Attribution Non Commercial (CC BY-NC 4.0) license, which permits others to distribute, remix, adapt, build upon this work non-commercially, and license their derivative works on different terms, provided the original work is properly cited, appropriate credit is given, any changes made indicated, and the use is non-commercial. See: http://creativecommons.org/licenses/by-nc/4.0/.

\section{ORCID iDs}

Roland Pastells-Peiró http://orcid.org/0000-0002-9561-9038
Carolina Climent-Sanz http://orcid.org/0000-0001-6911-2164

Montserrat Gea-Sánchez http://orcid.org/0000-0001-5143-3314

\section{REFERENCES}

1 World Health Organization. Proposed working definition of an older person in Africa for the MDS project. Geneva: World Health Organization, 2002. https://www.who.int/healthinfo/survey/ ageingdefnolder/en/

2 Eurostat. Population structure and ageing. Eurostat, 2019. https://ec. europa.eu/eurostat/statistics-explained/index.php?title=Population structure_and_ageing

3 Instituto Nacional de Estadística. Movimiento Natural de la Población (MNP). Indicadores Demográficos Básicos (IDB). Año 2019. Datos provisionales [Natural Population Movement (MNP). Basic Demographic Indicators (IDB). Year 2019. Provisional data]. Madrid: Instituto Nacional de Estadística, 2020. https://www.ine.es/prensa/ mnp_2019_p.pdf

4 European Comission. The 2015 ageing report. economic and budgetary projections for the 28 EU member states (2013-2060). 397. Luxembourg: Office of the European Union, 2015.

5 Echaves García A, Echaves C. Individualización o pluralización de modos de convivencia? Análisis de la realidad familiar en España mediante la evolución y características de los hogares jóvenes [Individualization or pluralization of modes of coexistence? Analysis of family reality in Spain through the evolution and characteristics of young households]. Revista Metamorfosis: Revista del Centro Reina Sofía sobre Adolescencia y Juventud 2018;9:130-53.

6 Instituto Nacional de Estadística. Encuesta continua de hogares. Año 2017 [Continuous household survey. Year 2017]. Madrid: Instituto Nacional de Estadística, 2018. https://www.ine.es/prensa/ech_2017. pdf

7 Envejecimiento en Red. Vivir solo... [Living alone...]. Madrid: Envejecimiento en red, 2020. http://envejecimientoenred.es/vivirsolos/

8 Mavrodaris A, Powell J, Thorogood M. Prevalences of dementia and cognitive impairment among older people in sub-Saharan Africa: a systematic review. Bull World Health Organ 2013;91:773-83.

9 Snir JA, Bartha R, Montero-Odasso M. White matter integrity is associated with gait impairment and falls in mild cognitive impairment. Results from the gait and brain study. Neuroimage Clin 2019;24:101975.

10 Arriola Manchola E, Carnero Pardo C, Freire Pérez A. Deterioro cognitivo leve en el adulto mayor. Documento de consenso [Mild cognitive impairment in the elderly. Consensus document]. Madrid: Sociedad Española de Geriatría y Gerontología, 2017.

11 OECD/EU. Health at a glance: Europe 2018: state of health in the EU cycle. 211. París: OECD Publishing, 2018.

12 Tomaskova H, Kuhnova J, Cimler R, et al. Prediction of population with Alzheimer's disease in the European Union using a system dynamics model. Neuropsychiatr Dis Treat 2016;12:1589-98.

13 Testimony of Harry Johns, president and CEO of the Alzheimer's Association. Fiscal year 2014 appropriations for alzheimer's-related activities at the U.S. Department of Health and Human Services. Subcommittee on Labor, Health and Human Services, Education and Related Agencies Committee on Appropriations United States House of Representatives. New York: Alzheimer's Association, 2013.

14 Villarejo Galende A, Eimil Ortiz M, Llamas Velasco S. Informe de la Fundación del Cerebro. Impacto social de la enfermedad de Alzheimer y otras demencias [Report by the Spanish Foundation of the Brain on the social impact of Alzheimer disease and other types of dementia]. Neurología 2017:1-11.

15 Fernández Cacho LM, MÁ GV, Laso Cavadas S. Enfermería y Salud 2.0: Recursos TICs en el ámbito sanitario [Nursing and Health 2.0: ICTs resources in the health sector]. Index de Enfermería 2016;25:51-5.

16 Millaruelo Trillo JM. Importancia de la implicación del paciente en EI autocontrol de SU enfermedad: paciente experto. Importancia de las nuevas tecnologías como soporte al paciente autónomo. Atención Primaria 2010;42:41-7.

17 TIC Salut Social. Transformación digital [Digital transformation]: TIC Salut Social,, 2020. Available: https://ticsalutsocial.cat/es/area/ transformacion-digital/

18 Institut d'Estadística de Catalunya. Use of computer and use of Internet. By frequency and age groups Barcelona: Institut d'Estadística de Catalunya, 2019. https://www.idescat.cat/pub/?id= aec\& $=617 \&$ lang $=e n$

19 Muntjir M, Rahul MD, Alhumyani H. An analysis of Internet of things (IoT): novel architectures, modern applications, security aspects 
and future scope with latest case studies. Int $\mathrm{J}$ Eng Res Technol 2017;6:422-48.

20 Verzijl D, Dervojeda K, Probst L. Internet of things. smart health. Internet market, industry, entrepreneurship and SMEs. Geneva: Business Innovation Observatory. European Comission, 2015

21 Forum WE. Industrial Internet of things. Unleashing the potential of connected products and services. 39. Geneva, 2015.

22 MAn AM, Bardallo Gay J, Domenech Sánchez JJ. Seguridad loT en sanidad. Estamos preparados? [IoT security in healthcare Are we ready?] 45. Madrid: APISA, 2018.

23 Moher D, Shamseer L, Clarke M, et al. Preferred reporting items for systematic review and meta-analysis protocols (PRISMA-P) 2015 statement. Syst Rev 2015;4:1

24 Harris AD, McGregor JC, Perencevich EN, et al. The use and interpretation of quasi-experimental studies in medical informatics. J Am Med Inform Assoc 2006;13:16-23.

25 Husebo BS, Heintz HL, Berge LI, et al. Sensing technology to monitor behavioral and psychological symptoms and to assess treatment response in people with dementia. A systematic review. Front Pharmacol 2019;10:1699.

26 Morris ME, Adair B, Ozanne E, et al. Smart technologies to enhance social connectedness in older people who live at home. Australas $J$ Age 2014;33:142-52.

27 McGowan J, Sampson M, Salzwedel DM, et al. PRESS Peer Review of Electronic Search Strategies: 2015 Guideline Statement. J Clin Epidemiol 2016;75:40-6.

28 EndNote [program]. X9.2 version. New York: Clarivate Analytics, 2020.

29 Covidence. Covidence 2019. Available: https://www.covidence.org/ home
30 de Morton NA. The PEDro scale is a valid measure of the methodological quality of clinical trials: a demographic study. Aust $J$ Physiother 2009;55:129-33.

31 GRADE Working Group. GRADE handbook. Handbook for grading the quality of evidence and the strength of recommendations using the GRADE approach. GRADE Working Group, 2013.

32 Higgins JPT, Green S, Cochrane C. Cochrane handbook for systematic reviews of interventions. Wiley-Blackwell, 2008.

33 Mavridis D, White IR. Dealing with missing outcome data in metaanalysis. Res Synth Methods 2020;11:2-13.

34 Ors Montenegro A, Laguna Pérez A. Reflexiones sobre EI envejecimiento Y La calidad de vida. Cultura de los Cuidados 1997;1:60-3.

35 Carstensen LL, Lockenhoff CE. Aging, emotion, and evolution: the bigger picture. Ann N Y Acad Sci 2003;1000:152-79.

36 Alvarado García AM, ÁM SM. Análisis del concepto de envejecimiento [Aging concept analysis]. Gerokomos 2014;25:57-62.

37 Mansilla ME. Etapas del desarrollo humano [Stages of human development]. Revista de Investigación en Psicología 2000;3:105-16.

38 Gustafson DH, McTavish F, Gustafson DH, et al. The effect of an information and communication technology (ICT) on older adults' quality of life: study protocol for a randomized control trial. Trials 2015;16:191.

39 Pinazo Hernandis S, Bellegarde Nunes MD. La soledad de las personas mayores. Conceptualización, valoración e intervención The loneliness of older people conceptualization, assessment and intervention]. Madrid 2018;176.

40 Hernández L, Calet N, Gonzalez-Lopez JA. Análisis acústico de la voz para La detección del deterioro cognitivo. Revista de Logopedia, Foniatría y Audiología 2020;40:67-76. 